\title{
Spectral method for thermal conductivity calculations
}

\author{
JU LI \\ Department of Materials Science and Engineering, Ohio State University, Columbus, OH 43210, USA \\ (e-mail:li.562@osu.edu)
}

Received 19 December 2004; Accepted 7 January 2005; Published online 11 May 2006

\begin{abstract}
We discuss several post-processing issues in molecular dynamics (MD) calculation of lattice thermal conductivity using the Green-Kubo formula. For crystals with high thermal conductivity such as $\mathrm{SiC}$, converting the MD raw data on heat current fluctuations into thermal conductivity result is non-trivial. One can accelerate the process using Fast Fourier Transform and the spectral method. A few mathematical subtleties are cleared up. Due to finite data length, integration of the correlation function must be terminated before noise takes over; two termination criteria are proposed which give reasonable results for crystalline $\beta$-SiC. A simple solvable model that illustrates the above points and can be used for code-checking is presented.
\end{abstract}

Keywords: Correlation function, Exponential fitting (EF), First-dip (FD) termination, Power spectrum, Termination criterion

\section{Introduction}

We have calculated the thermal conductivity tensor $\underline{\underline{\kappa}}$ of solid $\mathrm{SiC}$ with and without defects [1] using the Green-Kubo formula [2]

$$
\underline{\underline{\kappa}}=\frac{1}{k_{\mathrm{B}} T^{2} \Omega} \int_{0}^{\infty} d \tau\left\langle\mathbf{J}^{q}(0) \mathbf{J}^{q}(\tau)\right\rangle,
$$

where $\mathbf{J}^{q}$ is the instantaneous heat current of the system, provided by a molecular dynamics (MD) simulation run [3-6]. Details concerning the interatomic potential model, simulation method and results for this material can be found at the above reference. Our main topic in this paper is a technical aspect which is often not fully explained in the literature, that is how one evaluates $\underline{\kappa}$ once the MD simulation has been performed and a finite length of the $\mathbf{J}^{q}(t)$ data $\overline{\bar{h}}$ as been recorded.

The problem is not critical for thermal conductivity calculations in liquids and non-crystalline solids simply because the heat current correlation function

$$
\underline{\underline{g}}(\tau) \equiv \frac{1}{k_{\mathrm{B}} T^{2} \Omega}\left\langle\mathbf{J}^{q}(0) \mathbf{J}^{q}(\tau)\right\rangle
$$

has a rather short correlation time, as the heat carriers quickly get scattered. Thus, it is a relatively quick calculation which does not require very long simulation runs to get convergent results, and the correlation function even can be calculated on-the-fly. However, a perfect crystalline solid usually has a much longer correlation time, and thus a much higher thermal conductivity. ${ }^{1}$ 
In the case of crystalline $\beta$-SiC, even at the high temperature of $1500 \mathrm{~K}$, to get a barely convergent result of the correlation integral requires an MD run longer than 2 million timesteps $(800 \mathrm{ps})$. Further taking into the account that:

1. To get realistic results, an interatomic potential of some sophistication, in our case the Tersoff many-body potential [7,8], must be used.

2. The calculation of thermal conductivity or resistivity depends sensitively on the simulation cell size, due to the intrinsic three-phonon scattering contributions. In our work we find that a 216-particle cell is the minimum, while 512 or 1000 particle cells are recommended.

3. At lower temperatures, longer simulation runs are needed because the correlation time gets even longer. For instance, at $500 \mathrm{~K}$ a minimally convergent run is 8 million timesteps, or approximately $4000 \mathrm{ps}$.

4. We find that the results of a simulation run is sensitively dependent on the initial particle velocity (phonon density) distribution of the run, because when the crystal has a high thermal conductivity the existing phonons are not easily scattered; the system will retain 'memory' of the initial condition and does become effectively ergodic at least for some time. For this reason, we need to do several independent runs starting from different initial conditions, and average over the respective correlation functions.

the necessary computational effort is quite formidable. Kitagawa et al. [5] have reported similar constraints in calculating the thermal conductivity of AlN. Because of this, the treatment and analysis of the raw data become important issues.

The computation of $g(\tau)$ can be quite expensive. For instance, in the above case at $T=1500 \mathrm{~K}$ and $N=2 \times 10^{6}$ timesteps 'minimal run', the correlation in $g(\tau)$ persists

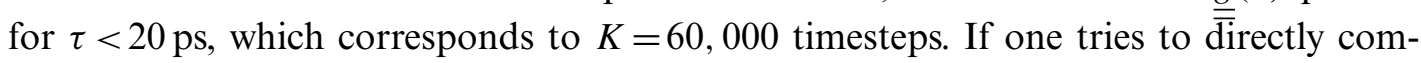
pute $g(\tau)$ by brute-force averaging, one faces multiplication operations on the order of $N \overline{\bar{K}}=10^{11}$, which means evaluating all tensorial components will take more than $2 \mathrm{~h}$ if the subroutine runs at $100 \mathrm{Mflop} / \mathrm{s}$. One can of course down-sample the data or decrease the number of time origins, but getting the raw data is an even more expensive process, so that one certainly does not want to waste any information in the post-processing stage. Furthermore, one is not sure what the correlation time is beforehand, and thus needs to see the entire $g(\tau)$.

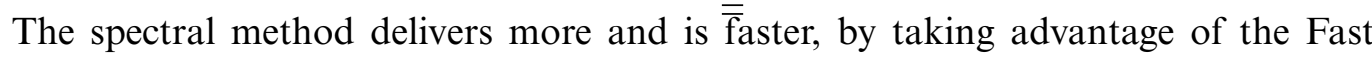
Fourier Transform (FFT) algorithm [9]. The number of operations is $\mathcal{O}(N \log N)$ with a small prefactor on the order of 10 . Because $\log N$ is always much smaller than $K$, it is faster than the brute-force method. It does not waste any information residing in the raw data, while also providing frequency-space information.

A flowchart of the procedure is shown in Figure 1. Basically one takes the FFT of the $\mathbf{J}^{q}\left(0<t<T_{f}\right)$ data to get $\mathbf{J}^{q}(\omega)$, square it and divide by $T_{f}$ to get the power spectrum $\underline{g}(\omega)$, and do an inverse FFT to get $\underline{g}(\tau)$. One can either directly read off the thermā conductivity by taking a limit of the power spectra

$$
\underline{\kappa}=\lim _{\omega \rightarrow 0} \frac{1}{k_{\mathrm{B}} T^{2} \Omega} \frac{\mathbf{J}^{q}(\omega) \mathbf{J}^{q *}(\omega)}{2 T_{f}}
$$

or take the more cautious route of integrating $\underline{\underline{g}}(\tau)$, whereas a termination of the integration is needed sooner or later due to finite data length. We shall propose two 


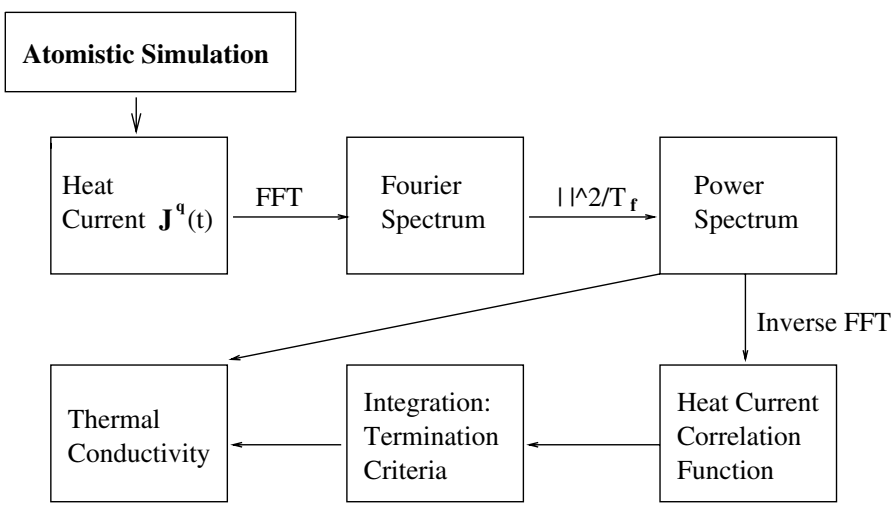

Figure 1. Flowchart of the spectral method to calculate the thermal conductivity.

termination criteria, applicable to crystalline $\mathrm{SiC}$, that minimize human intervention, yet giving reasonable results.

The spectral analysis of continuous variables will be introduced in Section 2. Its discrete implementation will be explained in Section 3, and the termination criteria for $\mathrm{SiC}$ will be explained in Section 4. A simple, analytically solvable model

$$
a_{i}=a_{i-1} e^{-\frac{1}{L}}+R_{i}
$$

is proposed in Section 5, which illustrates most of the above issues and can be used to test the code, hopefully providing a handle for beginners.

\section{Spectral analysis}

Consider a truncated function $f(t)$ which is finite from $t=0$ to $T_{f}$, and 0 elsewhere, so it is absolutely integrable. Then

$$
\begin{aligned}
F(\omega) & =\int_{-\infty}^{+\infty} f(t) \exp (i \omega t) d t, \\
f(t) & =\frac{1}{2 \pi} \int_{-\infty}^{+\infty} F(\omega) \exp (-i \omega t) d \omega .
\end{aligned}
$$

Notice that the artificial truncation of $f(t)$ will come to affect $F(\omega)$ statistically only when $\omega T_{f}$ is comparable to unity. In order to rule out the arbitrariness and make sure that the information we get is intrinsic to the signal, we should take care not to use the spectrum between $0<|\omega|<\pi / T_{f}$, which is of course a very small region because $T_{f}$ is large. Nevertheless, we cannot use $F(\omega)$ at $\omega$ equals absolute zero, because all our applications have finite $T_{f}$. We can only use $\omega_{0}$ 's which are much greater than $\pi / T_{f}$ but are still very small, as the limiting behavior of $\omega \rightarrow 0$.

The correlation function of $f(t)$ can be defined to be

$$
g(\tau)=\frac{1}{T_{f}} \int_{-\infty}^{+\infty} f^{*}(t) f(t+\tau) d t .
$$

For $g(\tau)$ to behave well, there should be $\tau \ll T_{f}$. Let's define a maximum correlation length $\tau_{\max }$ above which $g(\tau)$ is negligible; thus, $\tau_{\max } \ll T_{f}$. 
Plugging Eq. (4) into Eq. (5), there is

$$
\begin{aligned}
g(\tau)= & \frac{1}{4 \pi^{2} T_{f}} \int_{-\infty}^{+\infty} d \omega \int_{-\infty}^{+\infty} d \omega^{\prime} f^{*}(\omega) f\left(\omega^{\prime}\right) \exp \left(-i \omega^{\prime} \tau\right) \\
& \cdot \int_{-\infty}^{+\infty} \exp \left(i\left(\omega-\omega^{\prime}\right) t\right) d t \\
= & \frac{1}{4 \pi^{2} T_{f}} \int_{-\infty}^{+\infty} d \omega \int_{-\infty}^{+\infty} d \omega^{\prime} f^{*}(\omega) f\left(\omega^{\prime}\right) \exp \left(-i \omega^{\prime} \tau\right) \\
= & \frac{1}{2 \pi T_{f}} \int_{-\infty}^{+\infty} d \omega|F(\omega)|^{2} \exp (-i \omega \tau)
\end{aligned}
$$

which means that the Fourier spectrum of $g(\tau)$ is just the power spectrum of the signal $f(t):|F(\omega)|^{2} / T_{f}$.

There is a frequently cited result [4] that the integral of $g(\tau)$ from 0 to $+\infty$ is just the power spectrum $|F(\omega)|^{2} / T_{f}$ taken at $\omega=0$ and divided by 2 . This needs some clarification, because if we put $\omega=0$ in Eq. (3) directly, then

$$
F(\omega=0)=\int_{-\infty}^{+\infty} f(t) d t=\int_{0}^{T_{f}} f(t) d t
$$

which is actually a random number.

This is not what we expect. To see what is wrong, let

$$
A=\int_{-\tau_{\max }}^{\tau_{\max }} g(\tau) d \tau
$$

for a particular $\tau_{\max }$, then by Eq. (6),

$$
\begin{aligned}
A & =\frac{1}{2 \pi T_{f}} \int_{-\infty}^{+\infty} d \omega|F(\omega)|^{2} \int_{-\tau_{\max }}^{\tau_{\max }} \exp (-i \omega \tau) d \tau \\
& =\frac{1}{2 \pi T_{f}} \int_{-\infty}^{+\infty} d \omega|F(\omega)|^{2} \frac{2 \sin \left(\omega \tau_{\max }\right)}{\omega} .
\end{aligned}
$$

Since $T_{f}$ can be very large as we do long simulation runs, $\tau_{\max }$ can also be allowed to be large, provided that $\tau_{\max } \ll T_{f}$. Thus,

$$
\frac{2 \sin \left(\omega \tau_{\max }\right)}{\omega} \rightarrow 2 \pi \delta(\omega)
$$

as $\tau_{\max } \rightarrow+\infty$. Note that however, as we said before, we cannot really use the absolute $\omega=0$ value of $F(\omega)$, but only its limit well above $\pi / T_{f}$, so the system is able to demonstrate its intrinsic behavior in Eq. (3) through several oscillations. Also, notice that the main peak of Eq. (10) vanishes at $\pi / \tau_{\max }$, so we should pick

$$
\frac{\pi}{T_{f}} \ll\left|\omega_{0}\right|<\frac{\pi}{\tau_{\max }}
$$

such that $\omega_{0} T_{f} \gg 1$, yet $\omega_{0} \tau_{\max }<\pi$, and so

$$
A \approx \frac{1}{T_{f}}\left\langle\left|f\left(\omega_{0}\right)\right|^{2}\right\rangle
$$


by Eqs. (9) and (10), where \langle\rangle is taking the proper average over $\omega_{0}$ 's that satisfy Eq. (11). As both $T_{f}$ and $\tau_{\max }$ go to infinity with longer and longer MD runs and higher and higher accuracy requirement (while keeping $\tau_{\max } \ll T_{f}$ ), $\omega_{0} \rightarrow 0$, which is the true meaning of 'taking $\omega=0$ ' in $|F(\omega)|^{2} / T_{f}$.

For equilibrium systems, $g(-\tau)=g^{*}(\tau)$. If the signal is real, then $g(\tau)$ is also real, and we can write

$$
\int_{0}^{\tau_{\max }} g(\tau) d \tau \approx \frac{1}{2 T_{f}}\left\langle\left|f\left(\omega_{0}\right)\right|^{2}\right\rangle .
$$

\section{Discrete implementation}

FFT is the discrete implementation of Eqs. (3) and (4). Let

$$
f_{k}=f((k-1) \triangle), \quad \Delta=\frac{T_{f}}{N}, \quad k=1 . . N,
$$

then

$$
\begin{aligned}
F_{n} & =\sum_{k=1}^{N} f_{k} \exp \left(i \frac{2 \pi(n-1)(k-1)}{N}\right), \\
f_{k} & =\frac{1}{N} \sum_{n=1}^{N} F_{n} \exp \left(-i \frac{2 \pi(n-1)(k-1)}{N}\right) .
\end{aligned}
$$

Obviously, $F_{n}$ corresponds to $F(\omega=2 \pi(n-1) / N \Delta) / \Delta$ when $\omega \Delta \ll 1$, i.e., when the sample frequency is much larger than the characteristic frequency of the signal. In the case of lattice thermal conductivity, if we record the heat current at every MD timestep, then $\triangle$ is the MD step size, and the characteristic frequency of the heat current is limited by the highest-frequency phonon in the system. As a convention, $F_{0}$ is always set to zero by us after the FFT, because it represents the heat current average during the simulation, which should be subtracted off from the signal.

Let us define

$$
g_{j}=\frac{1}{N} \sum_{k=1}^{N} f_{k}^{*} f_{k+j-1}, \quad j=1 . . N,
$$

where $f_{k+j-1>N}$ is interpreted as $f_{k+j-1-N}$, and

$$
\begin{aligned}
G_{n} & =\sum_{j=1}^{N} g_{j} \exp \left(i \frac{2 \pi(n-1)(j-1)}{N}\right) \\
& =\frac{1}{N} \sum_{j, k=1}^{N} f_{k}^{*} f_{k+j-1} \exp \left(i \frac{2 \pi(n-1)(j-1)}{N}\right) \\
& =\frac{1}{N}\left|F_{n}\right|^{2} .
\end{aligned}
$$


And so,

$$
g_{j}=\frac{1}{N} \sum_{n=1}^{N} G_{n} \exp \left(-i \frac{2 \pi(n-1)(j-1)}{N}\right) .
$$

The idea is that we can calculate the correlation function by first FFT the original signal $f_{k}$, take the square module of it and divide by $N$, then inverse FFT back.

The discrete power spectrum summation

$$
\frac{\left|F_{n}\right|^{2}}{N}=\frac{1}{N} \sum_{k, k^{\prime}=1}^{N} f_{k} f_{k^{\prime}}^{*} \exp \left(i \frac{2 \pi(n-1)\left(k^{\prime}-k\right)}{N}\right)
$$

is represented graphically in Figure 2. The discretized maximum correlation length is $K=N \tau_{\max } / T_{f}$. The shaded region lies between $\left|k-k^{\prime}\right|<K$, so $f_{k}, f_{k^{\prime}}$ are correlated within. Outside the shaded region their products can be regarded as random noise, so passing through a wave with enough many oscillations in the range would filter them out, which then requires

$$
\frac{2 \pi n}{N} N=2 \pi n \gg 2 \pi \text {. }
$$

We may also want to sum up the correlation $f_{k} f_{k^{\prime}}^{*}$ inside the shaded region, which requires the phase factor $\exp \left(i 2 \pi(n-1)\left(k^{\prime}-k\right) / N\right)$ in Eq. (19) to be approximately unity for $\left|k-k^{\prime}\right|<K$. Thus, if

$$
\frac{2 \pi n}{N} K \ll \frac{\pi}{2}
$$

or combined with Eq. (20),

$$
1 \ll n \ll N / 4 K
$$

we would have

$$
\frac{\left|F_{n}\right|^{2}}{N} \approx 2\left\langle\sum_{j=1}^{K} f_{1} f_{j}\right\rangle
$$

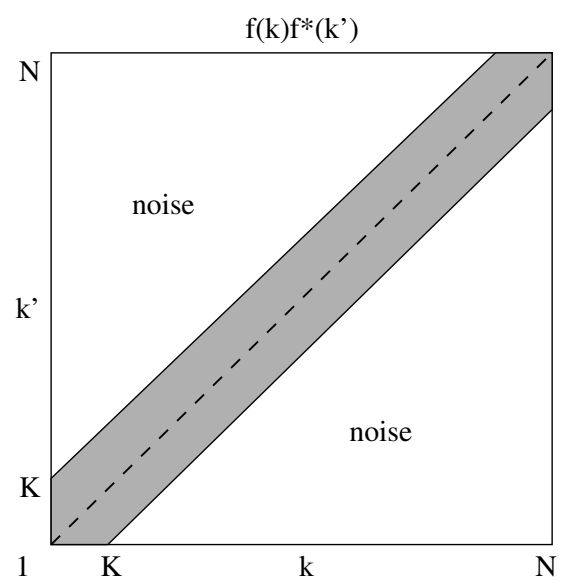

Figure 2. Discrete power spectrum summation. 
It is easy to see that finding such an $n$ is equivalent to choosing $\omega_{0}$ in the case of continuous signal.

\section{Simulation results and correlation integral termination}

From now on we will properly redefine the heat current: $\mathbf{J}^{q}(t) \rightarrow \mathbf{J}^{q}(t) / \sqrt{k_{\mathrm{B}} T^{2} \Omega}$, such that the $1 / k_{\mathrm{B}} T^{2} \Omega$ factor in Eq. (1) vanishes. Also, $\tau$ will take the unit of pico-seconds and $\underline{\kappa}$ will take the unit of $\mathrm{W} / \mathrm{m} / \mathrm{K}$ by default. The spectral analysis of $f(t)$ can be easily generalized to vector $\mathbf{J}^{q}(t)$ whereby we define

$$
g_{i j}(\tau) \equiv\left\langle J_{i}^{q}(0) J_{j}^{q}(\tau)\right\rangle,
$$

so there is

$$
\kappa_{i j}=\int_{0}^{\infty} d \tau g_{i j}(\tau)
$$

$\mathbf{J}^{q}(t)$ and $g_{i j}(\tau)$ are real. Furthermore, by time-reversal symmetry of physical systems, $g_{i j}(\tau)=g_{i j}(-\tau)$. So we should have

$$
\begin{aligned}
g_{i j}(\tau) & =\frac{1}{2 \pi T_{f}} \int_{-\infty}^{+\infty} d \omega\left\langle J_{i}^{*}(\omega) J_{j}(\omega)\right\rangle \exp (-i \omega \tau) \\
& =\frac{1}{2 \pi T_{f}} \int_{-\infty}^{+\infty} d \omega \operatorname{Re}\left[\left\langle J_{i}^{*}(\omega) J_{j}(\omega)\right\rangle\right] \exp (-i \omega \tau) .
\end{aligned}
$$

For crystals with point-group symmetry no lower than $\mathrm{T}_{\mathrm{d}}$, such as $\beta$-SiC, $\underline{\kappa}$ is a scalar matrix: $\kappa_{i j}=\kappa \delta_{i j}$, and we can use this symmetry by defining $|J(\omega)|^{2}=$ $\left(\left|J_{x x}^{q}(\omega)\right|^{2}+\left|J_{y y}^{q}(\omega)\right|^{2}+\left|J_{z z}^{q}(\omega)\right|^{2}\right) / 3$ and $g(\tau)=\left(g_{x x}+g_{y y}+g_{z z}\right) / 3$, from the data of a single MD run. So $\kappa=\left(\kappa_{x x}+\kappa_{y y}+\kappa_{z z}\right) / 3$.

Theoretically, the power spectrum $|J(\omega)|^{2} / 2 T_{f}$ provides complete information about the thermal conductivity, because

$$
\begin{aligned}
\kappa & =\int_{0}^{+\infty} g(\tau) d \tau \\
& =\lim _{\omega_{0} \rightarrow 0} \frac{1}{2 T_{f}}\left|J\left(\omega_{0}\right)\right|^{2} .
\end{aligned}
$$

In practice, however, it is not straightforward to implement because $|J(\omega)|^{2} / 2 T_{f}$ from a single MD run is usually a 'cloud' of points which requires smoothing. Figure 3 shows such a power spectrum from a 2 million timestep MD simulation of a $\beta$-SiC crystal in a 512-particle cell at $1500 \mathrm{~K}$. The $x$-axis is the frequency number $n$ after FFT. The $y$-axis is properly scaled to the thermal conductivity unit $\mathrm{W} / \mathrm{m} / \mathrm{K}$. The raw values are plotted in empty circles, while results after properly smoothing are shown in dots with solid line to guide the eye. As we show in the last section, taking $\omega_{0}$ to zero in Eq. (27) is equivalent to choosing an $n$ on the discrete grid such that $1 \ll$ $n \ll N / 4 K$. We shall see in Figure 4 that the correlation $\langle J(0) J(\tau)\rangle$ is significant for $\tau<\tau_{\max } \approx 20 \mathrm{ps}$, which corresponds to $K=60,000$. So $n$ should satisfy $1 \ll n \ll 10$, say $n=6$ (in this case because $|J(\omega)|^{2}$ is the average of three independent directions, the bounds are not so strict), which roughly corresponds in Figure 3 to a thermal 


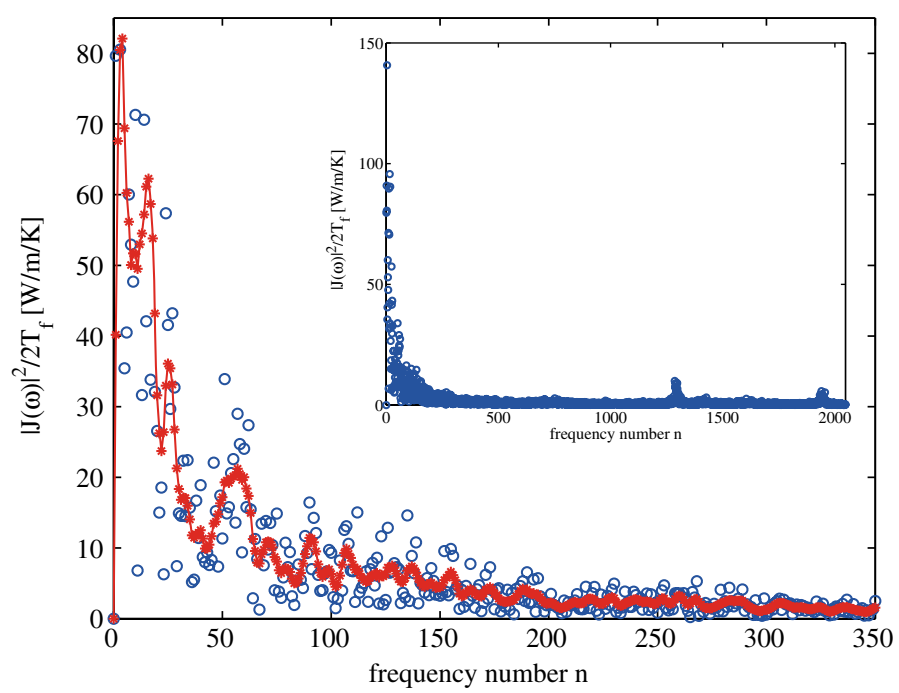

Figure 3. Power spectrum (plotted in circles and properly normalized to the unit of $\mathrm{W} / \mathrm{m} / \mathrm{K}$ ) versus frequency number $n$, of the heat current data from a 747 ps MD simulation of $\beta$-SiC crystal at $T=1500 \mathrm{~K}$ in a 512-particle cell. The dots are results after 4-point smoothing, with solid line to guide the eye.

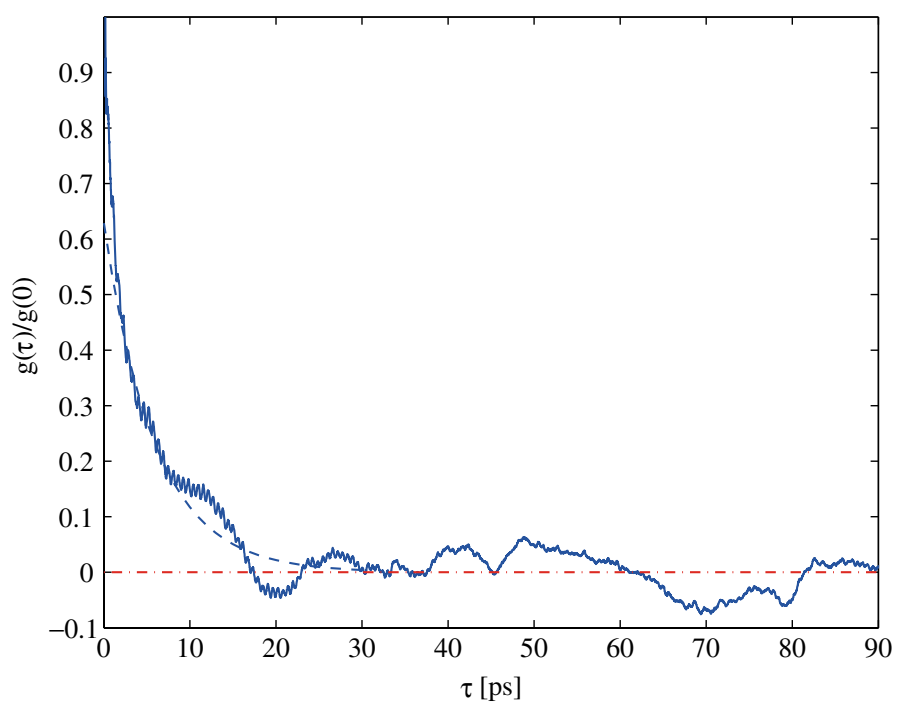

Figure 4. Normalized heat current correlation function $g(\tau) / g(0)$ from a single MD run $(g(0)=17.3$ $\mathrm{W} / \mathrm{m} / \mathrm{K} / \mathrm{ps}$ ), for perfect $\beta$-SiC at $1500 \mathrm{~K}$. The dashed line is the fit to exponential decay in the range $1-9$ ps.

conductivity value of $70 \pm 15 \mathrm{~W} / \mathrm{m} / \mathrm{K}$, in reasonably good agreement with the experimental value of $62.6 \mathrm{~W} / \mathrm{m} / \mathrm{K}$. However, this is probably the most we can see from the power spectrum; the indeterminate nature of this inference procedure forces us to find better, or at least more automatic, ways of finding out the simulation thermal conductivity result.

Notice that the requirement Eq. (22) is only barely satisfied in the above example due to the smallness of $N / 4 K$, which is the reason we call this a minimal run. As 
we shall demonstrate later, the error bar of one's result is largely determined by the ratio $N / 4 K$. By doing longer simulation runs at the same temperature, one increases $N / 4 K$ and thus improves the quality of the final thermal conductivity result.

As a brief digression, we note (see inset of Figure 3) that the power spectra sometimes show small peaks at finite frequencies. Besides roughening up $g(\tau)$ a bit, this will have little influence on the calculated thermal conductivity. Nevertheless, the nature of these peaks, whether being something physical or as a simulation artifact such as finite cell size effect, is unknown.

We now turn to the correlation function picture by inverse Fourier transforming $|J(\omega)|^{2}$ using Eq. (18). Figure 4 shows in solid line the normalized correlation function $g(\tau) / g(0)$ after proper local smoothing. Only the first $1 / 8$ portion of the entire data is plotted in the graph (total run length $=747 \mathrm{ps}$ ) because it appears to be completely random fluctuations after that. Clearly, we need a termination criterion if somehow we want to retrieve the $\kappa=\int_{0}^{+\infty} g(\tau) d \tau$ information. Figure 4 represents the typical outcome of a minimal run, which provides a test case for the termination criterion.

One observation is that in the case of $\beta$-SiC, especially at high temperatures, the actual shape of $g(\tau)$ closely resembles that of an exponential decay with somewhat faster relaxation in the first few pico-seconds and a slight flattening of the tail. This observation is confirmed by simulation results after multiple runs are performed and better statistics are collected (see Figures 5-7). It leads us to propose the following exponential fitting (EF) criterion. We can do a least square fit of $g(\tau)$ to an exponential decay form $a e^{-\left(\tau-\tau_{f}\right) / b}$ in the time range $\left[\tau_{i}, \tau_{f}\right]$, where simulation accuracy is still believed to be good, in order to determine the best coefficients $\{a, b\}$. We then numerically integrate $g(\tau)$ up to $\tau_{f}$, and add the analytically estimated tail contribution $a b$. This termination rule only requires two parameters to be chosen beforehand, $\tau_{i}$ and $\tau_{f}$, depending on simulation run length, correlation time and number of runs being averaged. The recommended values for $\beta$-SiC are given in Table 1, along with other simulation parameters.

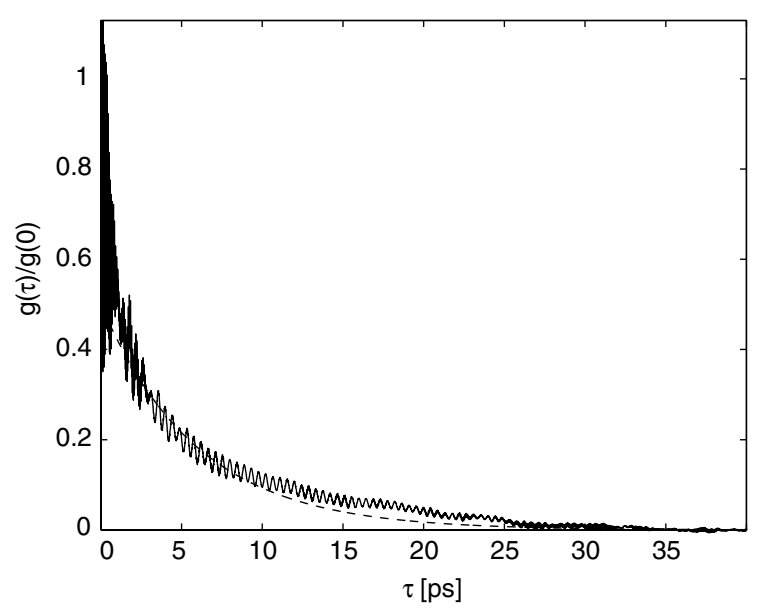

Figure 5. Well-converged normalized heat current correlation function $g(\tau) / g(0)$ for perfect $\beta$-SiC at $1700 \mathrm{~K}(g(0)=22.0 \mathrm{~W} / \mathrm{m} / \mathrm{K} / \mathrm{ps})$. The dashed line is the fit to exponential decay in the range $1-9 \mathrm{ps}$. 


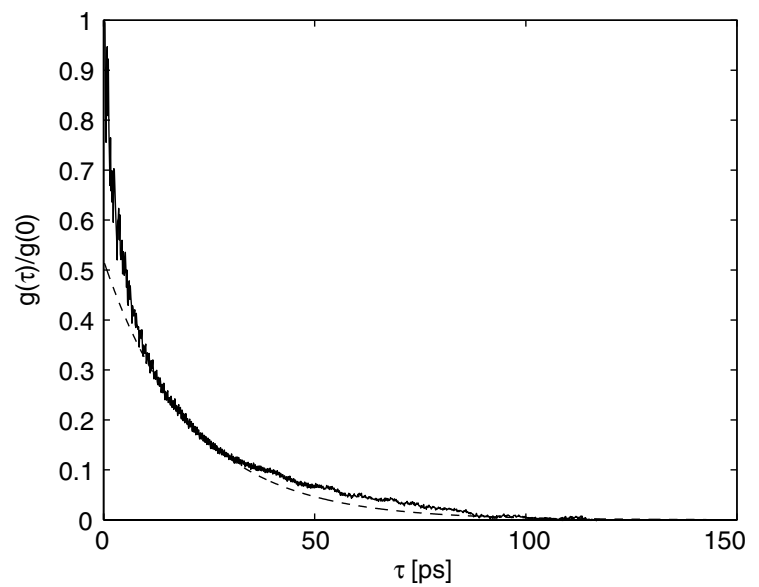

Figure 6. Well-converged normalized heat current correlation function $g(\tau) / g(0)$ for perfect $\beta$-SiC at $760 \mathrm{~K}(g(0)=15.5 \mathrm{~W} / \mathrm{m} / \mathrm{K} / \mathrm{ps})$. The dashed line is the fit to exponential decay in the range 5-25 ps.

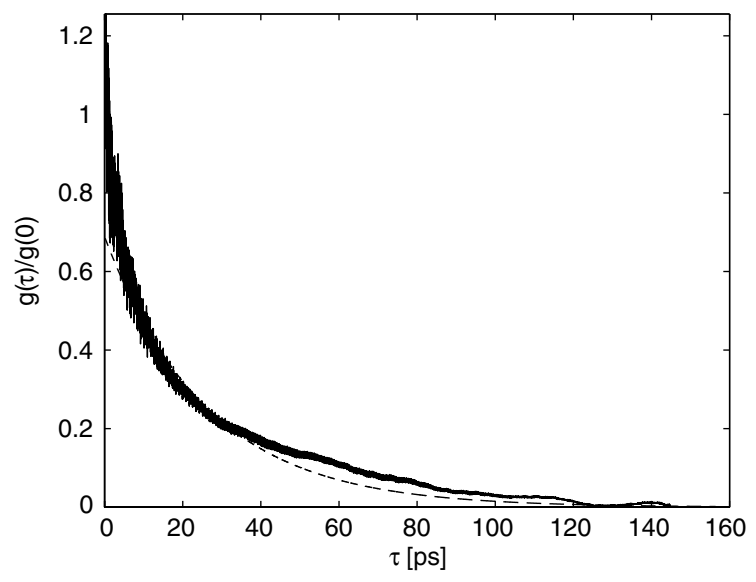

Figure 7. Well-converged normalized heat current correlation function $g(\tau) / g(0)$ for perfect $\beta$-SiC at $284 \mathrm{~K}(g(0)=7.8 \mathrm{~W} / \mathrm{m} / \mathrm{K} / \mathrm{ps})$. The dashed line is the fit to exponential decay in the range 5 to 35 ps.

Table 1. Recommended 'minimal-run' parameters for calculating $\beta$-SiC perfect crystal lattice thermal conductivity

\begin{tabular}{llllll}
\hline \multicolumn{1}{c}{$T(\mathrm{~K})$} & Steps & Timestep $(\mathrm{fs})$ & Runs & $\mathrm{EF} \tau_{i}(\mathrm{ps})$ & $\mathrm{EF} \tau_{f}(\mathrm{ps})$ \\
\hline $250-500$ & $8 \times 10^{6}$ & $0.30-0.35$ & $6-10$ & 5.0 & 35.0 \\
$500-1000$ & $4 \times 10^{6}$ & $0.30-0.35$ & $4-6$ & 3.5 & 21.0 \\
$1000-1500$ & $4 \times 10^{6}$ & $0.25-0.30$ & $3-4$ & 1.0 & 9.0 \\
$>1500$ & $2 \times 10^{6}$ & $<0.25$ & $3-4$ & 1.0 & 9.0 \\
\hline
\end{tabular}

Another more straightforward way is to postulate that the actual heat current correlation function $g(\tau)$ never turns negative. We know that certain self-correlation functions such as the velocity autocorrelation function in liquids can have negative parts sometimes, but the occurrence is rare and is unlikely to happen in solid SiC. If this is the case, then at the point where the calculated correlation curve first turns 
negative, one can argue that the calculated curve must be finally 'corrupted' by random fluctuations, and the data from there on cannot be trusted. One can then estimate the thermal conductivity by numerically integrating the raw data up to the point where the 'first dip' happens. We will refer to this as the first-dip (FD) rule.

Usually $g(\tau)$ from a long simulation run with a large $N / 4 K$ ratio or the average of multiple runs will give FD and EF results that are close to each other, especially at high temperatures, while large difference occurs for those runs that are not well-converged (small $N / 4 K$ ) and can be seen to be rather irregular. We list several examples down below:

- 2 million step run, $T=1500 \mathrm{~K}, 512$-particle cell (see Figures 3 and 4). EF result: $71.2 \mathrm{~W} / \mathrm{m} / \mathrm{K}$; FD result: $67.8 \mathrm{~W} / \mathrm{m} / \mathrm{K}$; Exp't: $62.6 \mathrm{~W} / \mathrm{m} / \mathrm{K}$.

- 2 million step run, $T=1055 \mathrm{~K}, 216$-particle cell. EF result: $88.6 \mathrm{~W} / \mathrm{m} / \mathrm{K}$; FD result: $89.3 \mathrm{~W} / \mathrm{m} / \mathrm{K}$; Exp't: $95.7 \mathrm{~W} / \mathrm{m} / \mathrm{K}$.

- 2 million step run, $T=790 \mathrm{~K}, 216$-particle cell. EF result: $172.6 \mathrm{~W} / \mathrm{m} / \mathrm{K}$; FD result: $229.7 \mathrm{~W} / \mathrm{m} / \mathrm{K}$; Exp't: $162.4 \mathrm{~W} / \mathrm{m} / \mathrm{K}$.

- Average of $4 \times 4$ million step runs (see Figure 5), $T=1700 \mathrm{~K}, 216$-particle cell. EF result: $69.2 \mathrm{~W} / \mathrm{m} / \mathrm{K}$; FD result: $77.5 \mathrm{~W} / \mathrm{m} / \mathrm{K}$; Exp't: $51.9 \mathrm{~W} / \mathrm{m} / \mathrm{K}$.

- Average of $4 \times 4$ million step runs (see Figure 6), $T=760 \mathrm{~K}, 216$-particle cell. EF result: $152.2 \mathrm{~W} / \mathrm{m} / \mathrm{K}$; FD result: $162.7 \mathrm{~W} / \mathrm{m} / \mathrm{K}$; Exp't: $138.5 \mathrm{~W} / \mathrm{m} / \mathrm{K}$.

- Average of $12 \times 8$ million step runs (see Figure 7), $T=284 \mathrm{~K}, 216$-particle cell. EF result: $146.2 \mathrm{~W} / \mathrm{m} / \mathrm{K}$; FD result: $161.3 \mathrm{~W} / \mathrm{m} / \mathrm{K}$; Exp't: $318.6 \mathrm{~W} / \mathrm{m} / \mathrm{K}$.

Just by looking at the results and comparing with experiments, one cannot clearly distinguish between the error due to the model, such as inexact interatomic potential or the treatment of quantum effects which is quite crude at low temperatures [1], and the error due to the implementation, such as the termination criteria. They can only be distinguished after doing many identical runs with different random seeds, and accumulating enough data so that we can claim certain result to be the true simulation result, and then benchmark the quality of a specific implementation by feeding it only part of the raw data available. This procedure is very time-consuming. From experience, we propose that for a single minimal run, the EF result may be more accurate because it acts like a filter; but when one can manage to do long simulation runs, and many of them, the FD rule may yield better results because one then knows the tail part to be real. Table 1 lists the parameters which we think are appropriate for $\beta$-SiC crystal at various temperatures.

We note that the difficulty in benchmarking implementations does not exist if we know the exact correlation function. This is done in Section 5 where we propose a simple analytically solvable model.

\section{Simple solvable model}

Consider discrete data series

$$
a_{0}=0, \quad a_{i}=a_{i-1} e^{-1 / L}+R_{i},
$$

where $R_{i}$ is an independent random number uniformly distributed on $(-0.5,0.5)$, and $L$ is an arbitrary constant signifying the characteristic correlation length. The series can be shown to have a simple correlation function: 


$$
\begin{aligned}
g_{k} & =\left\langle a_{i} a_{i+k-1}\right\rangle \\
& =\left\langle a_{i} a_{i+k-2} e^{-1 / L}+a_{i} R_{i+k-1}\right\rangle \\
& =\left\langle a_{i} a_{i+k-2}\right\rangle e^{-1 / L},
\end{aligned}
$$

because $R_{i+k-1}$ is not correlated with $a_{i}$. And so

$$
g_{k}=\left\langle a_{i} a_{i}\right\rangle e^{-(k-1) / L} .
$$

Now,

$$
\begin{aligned}
a_{i} & =R_{i}+a_{i-1} e^{-1 / L} \\
& =R_{i}+R_{i-1} e^{-1 / L}+R_{i-2} e^{-2 / L}+\cdots
\end{aligned}
$$

so

$$
\begin{aligned}
\left\langle a_{i} a_{i}\right\rangle & =\left\langle R_{i}^{2}\right\rangle+\left\langle R_{i-1}^{2}\right\rangle e^{-2 / L}+\left\langle R_{i-2}^{2}\right\rangle e^{-4 / L}+\cdots \\
& =\frac{\left\langle R^{2}\right\rangle}{1-e^{-\frac{2}{L}}} .
\end{aligned}
$$

Because

$$
\left\langle R^{2}\right\rangle=\int_{-0.5}^{0.5} x^{2} d x=\frac{1}{12},
$$

there is

$$
\left\langle a_{i}^{2}\right\rangle=\frac{1}{12\left(1-e^{-\frac{2}{L}}\right)},
$$

and

$$
g_{k}=\frac{e^{-(k-1) / L}}{12\left(1-e^{-\frac{2}{L}}\right)} .
$$

The theoretical thermal conductivity is then

$$
\kappa \equiv \sum_{k=1}^{+\infty} g_{k}=\frac{1}{12\left(1-e^{-\frac{2}{L}}\right)\left(1-e^{-\frac{1}{L}}\right)} .
$$

Using this known result we can check our codes by directly plugging in the series, and see if after the procedure in Figure 1, we indeed get a correlation function of the correct form and a correct 'thermal conductivity' value. This is the best way to ensure that all the coefficients are made right.

Let us illustrate the issues discussed in this paper by numerically studying three examples of Eq. (28), following the steps outlined in Figure 1:

- Case A: $N=2^{21}, N / L=1000$, representing a well-converged MD run. The theoretical thermal conductivity is $\kappa_{A}=1.834 \times 10^{5}$.

- Case B: $N=2^{21}, N / L=100$, representing a minimal MD run. ${ }^{2}$ The theoretical thermal conductivity should be $\kappa_{B}=1.833 \times 10^{7}$.

- Case C: Identical to case B but with a different random number seed. 


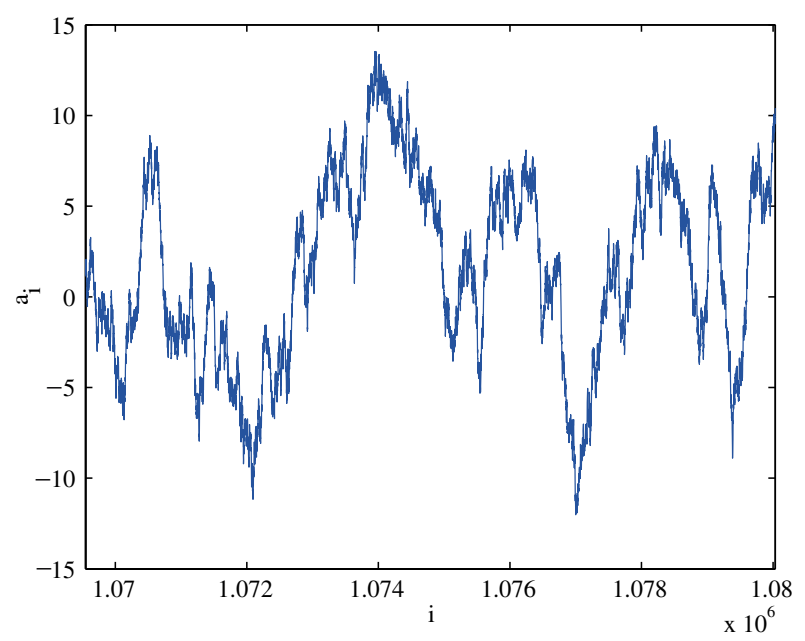

Figure 8. A small portion of the random signal $a_{i}$ in case A, with $N=2^{21}$ and $N / L=1000$.

where we take care to let the sequence iterate $N / 8$ steps to 'equilibrate' before collecting the $N$ data points. Figure 8 shows a small portion of the signal in case A for $5 L \approx 10,000$ steps.

Figure 9 plots the power spectrum $\left|F_{n}\right|^{2} / 2 N$ of case $\mathrm{A}$ (the inset shows the raw data on a larger scale) versus the frequency number $n$ between $[1, N / L]$, which are the only relevant ones to thermal conductivity as we demonstrated in Section $3^{2}$. The empty circles are the raw values, while the dots are the smoothed result after passing through a zero-phase filter of nearby 10 points $\left(\right.$ MATLAB ${ }^{\circledR}$ filtfilt), with solid line to guide the eye. We can see that the raw data is still a 'cloud' of points, very much like that of Figure 3, but after smoothing it becomes more regular, with discernible leveling off as $n \rightarrow 0$, which is related to the fact that as $L \gg 1,\left|F_{n}\right|^{2} / 2 N$ becomes a Lorentzian. The $n \rightarrow 0$ limit, as we can see, corresponds to a thermal conductivity value of about $(1.7 \pm 0.3) \times 10^{5}$ at frequency number $n$ around $N / 25 L$. This verifies Eq. (23) and Eq. (22) and the agreement is actually pretty good for this 'well-converged run' with $N / L=1000$.

We then do inverse FFT of the power spectrum to get $g_{k}$. The numerical result is plotted in Figure 10 in solid line, against the theoretical prediction of Eq. (34) in dashed line. Since the only timescale in this problem is $L$, the $x$-axis is scaled as $k / L$. The numerical result is also shown in the inset on a larger scale. We can clearly discern a region $(5<k / L<8)$ in the numerical result where $g_{k} \approx 0$ and is almost flat and parallel to the $x$-axis, to the left of which $g_{k}$ is regular and well-converged, and to the right it begins to randomly fluctuate. Due to the existence of this region, the thermal conductivity result we get using $g_{k}$ (versus the power spectrum method) is bound to be very good, no matter which termination rule is invoked. And this is what actually happens to defect-state $\mathrm{SiC}$ thermal conductivity calculations, where the correlation times drop by a decade or so compared to the perfect crystal at the same temperature [1].

We then apply our EF rule to the data: the region of fitting $\left(\left[\tau_{i}, \tau_{f}\right]\right.$ in last section) is chosen to be $k / L=[0.5,1.5]$ (indicated by the two dots) which roughly corresponds to same position in $\beta$-SiC. The resultant exponential curve is plotted in dotted lines, 


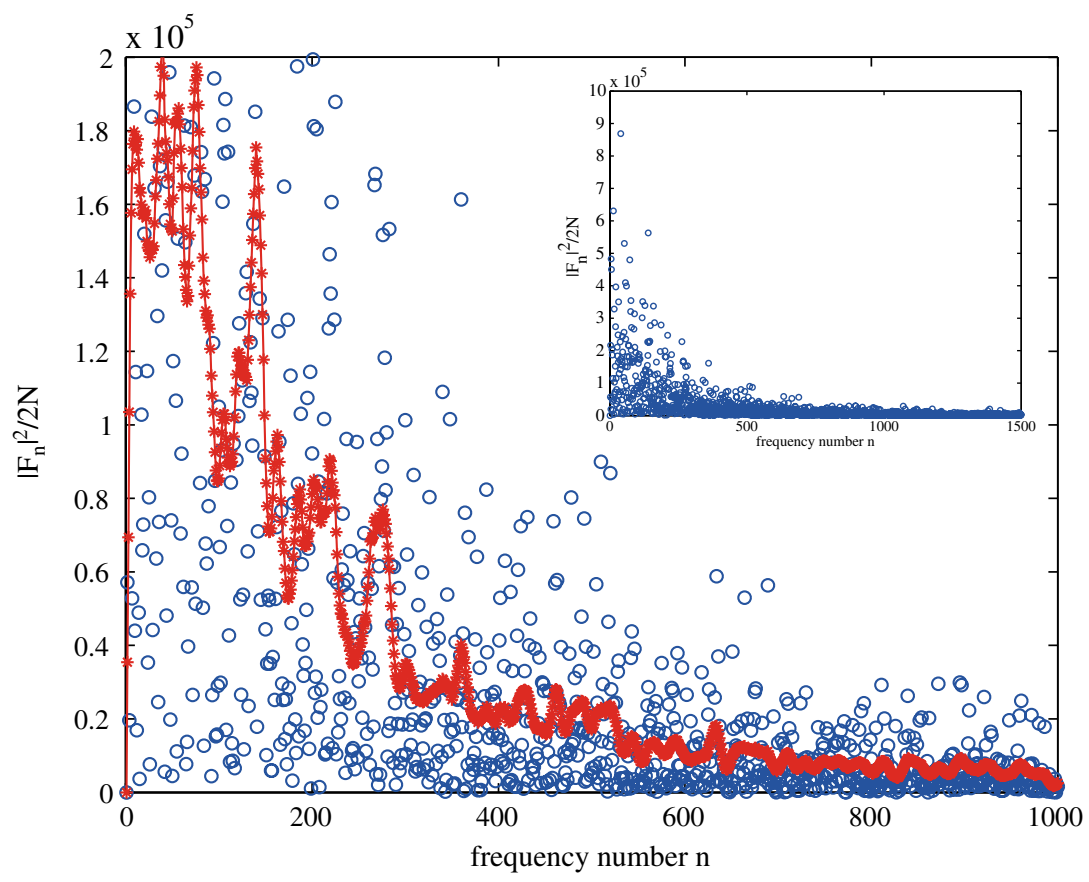

Figure 9. Power spectrum $\left|F_{n}\right|^{2} / 2 N$ versus frequency number $n$ in case A. The empty circles are the raw data, while the dots (with solid line to guide the eye) are the smoothed results after passing through a zero-phase filter of nearby 10 points. The inset graph shows the raw data on a larger scale.

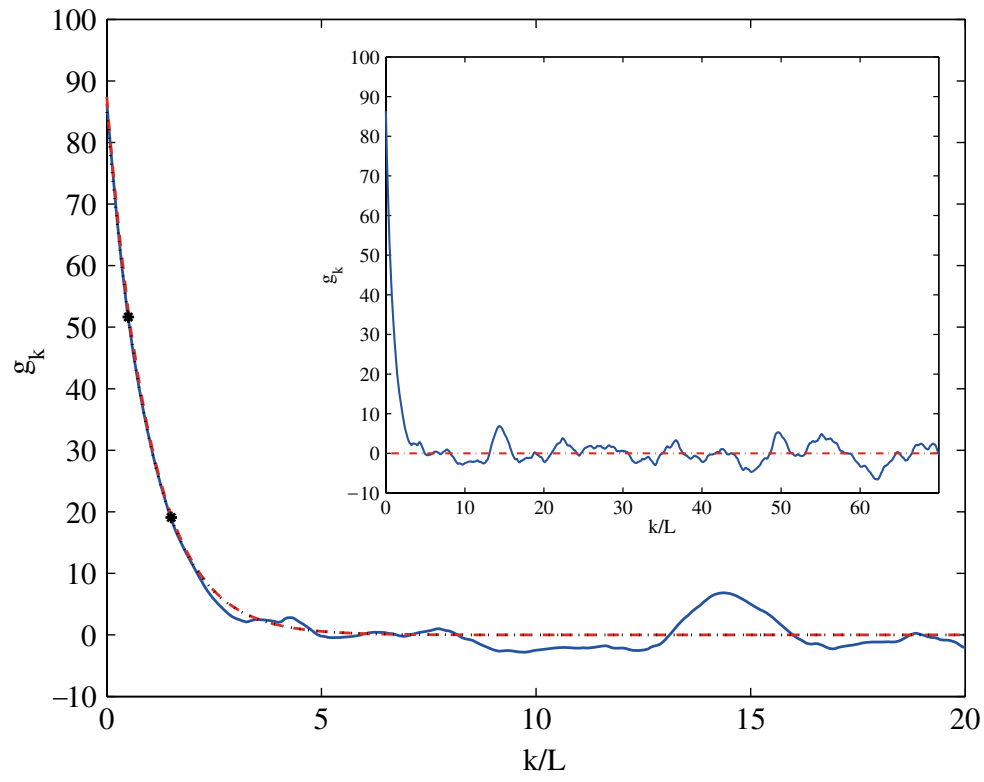

Figure 10. Correlation function $g_{k}$ versus $k / L$ in case A. The solid line is the numerical result; the dashed line is the theoretical prediction of Eq. (34). The dotted line (indistinguishable from the theoretical curve in this case) is the EF fit to numerical result in the region $0.5 \leq k / L \leq 1.5$, indicated by the two dots. The inset graph shows the numerical result on a larger scale. 
which because it has the correct functional dependence, is almost indistinguishable from the theoretical curve. The inferred characteristic decay time is $L^{\prime} / L=1.0043$, and the inferred thermal conductivity is $\kappa_{A}^{\mathrm{EF}} / \kappa_{A}=0.979$.

If we apply the FD rule to the same data, we find that the first dip occurs at $k_{\text {dip }} / L=4.918$, which of course is completely random, and the FD estimation gives a thermal conductivity value of $\kappa_{A}^{\mathrm{FD}} / \kappa_{A}=0.962$.

The entire procedure for case A registers $2.75 \times 10^{8}$ flops in MATLAB ${ }^{\circledR}$ while the brute-force method requires computations on the order of $N L=4.40 \times 10^{9}$ flops, thus demonstrating the efficiency of this approach. The comparison of cost will go even more favorably for the spectral method in cases B and C, which represent more realistic scenarios. Furthermore, the spectral method loses no information about the original data, and one can gain extra knowledge about the power spectrum.

Now let us do case B. The meaning of symbols in the figures will be the same as those in case A. Figure 11 shows the power spectrum, where the dots are the smoothed result of nearby 5 points. Because $N / L$ is small, we have much less relevant frequency numbers than those in Figure 11. The $n \rightarrow 0$ limit gives a thermal conductivity value of $(2 \pm 0.6) \times 10^{7}$ around $n=7$, which agrees well with the theoretical value of $\kappa_{B}=1.833 \times 10^{7}$, considering this is a minimal run.

Figure 12 shows the correlation function $g_{k}$ versus $k / L$. We still fit the numerical result to an exponential form in the region $0.5 \leq k / L \leq 1.5$. The inferred characteristic decay time is $L^{\prime} / L=1.280$, and the inferred thermal conductivity is $\kappa_{B}^{\mathrm{EF}} / \kappa_{B}=1.518$. The fitted exponential curve is shown in dotted lines, where the region of fitting is

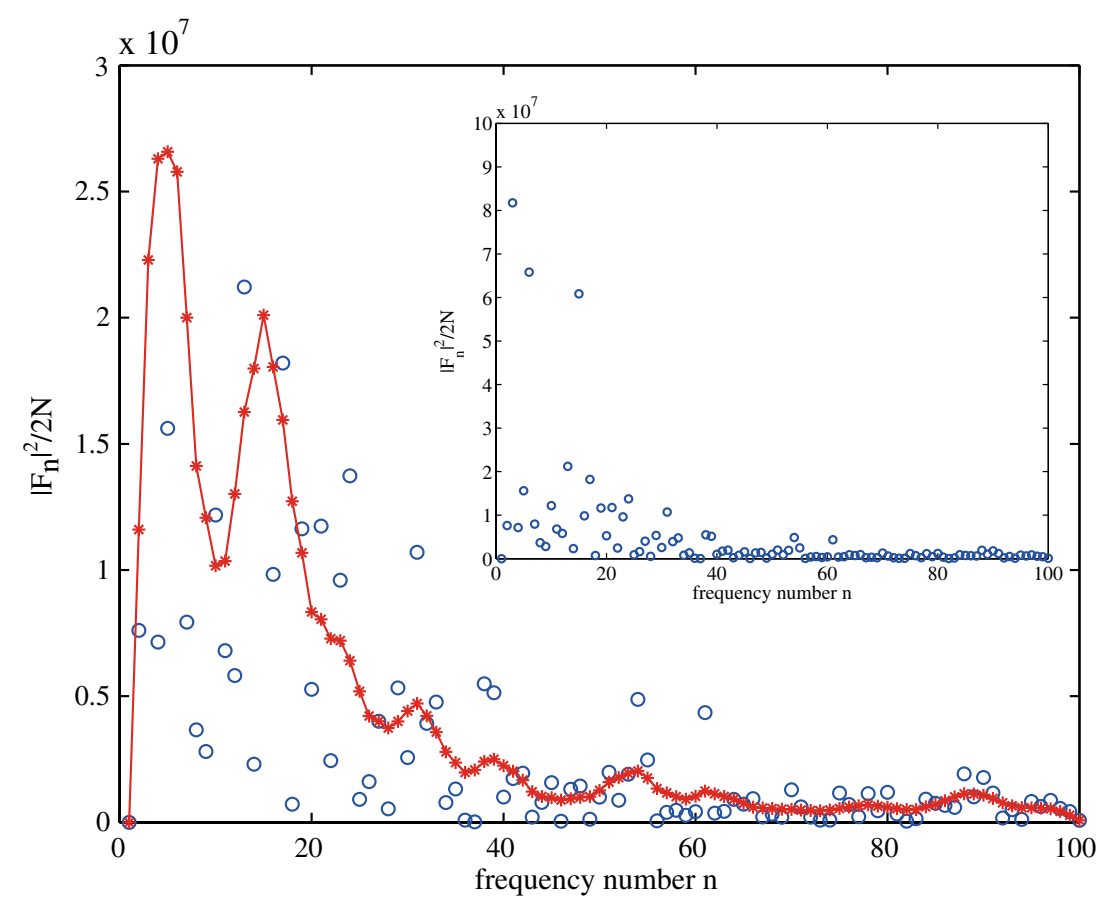

Figure 11. Power spectrum $\left|F_{n}\right|^{2} / 2 N$ versus frequency number $n$ in case B. The empty circles are the raw data, while the dots (with solid line to guide the eye) are the smoothed results after passing through a zero-phase filter of nearby 5 points. The inset graph shows the raw data on a larger scale. 


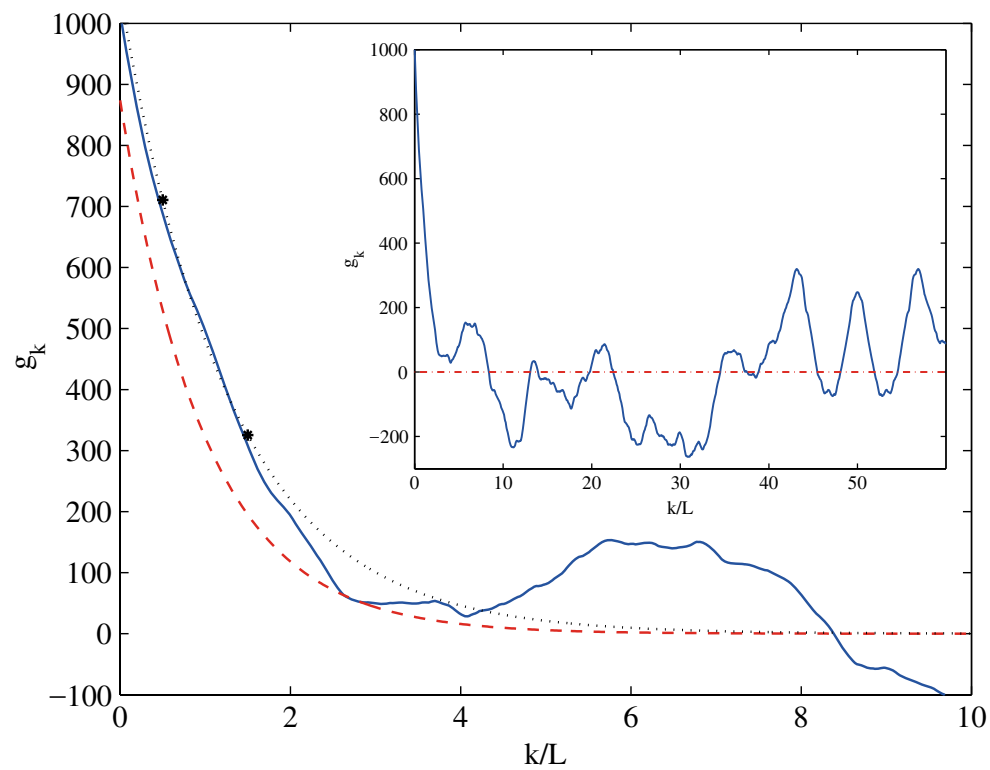

Figure 12. Correlation function $g_{k}$ versus $k / L$ in case B. The solid line is the numerical result; the dashed line is the theoretical prediction of Eq. (34). The dotted line is the EF fit to numerical result in the region $0.5 \leq k / L \leq 1.5$, indicated by the two dots. The inset graph shows the numerical result on a larger scale.

between the two dots. We can see that neither the original data nor the EF curve agree well with the theoretical curve, due to small $N / L$, i.e., insufficient data. However, the EF curve is able to filter out the fictitious bulge between $4<k / L<8$.

If we apply the FD rule to the same data, we find that in this case the first dip happens at $k_{\mathrm{dip}} / L=8.385$, and the FD estimation of the thermal conductivity value is $\kappa_{A}^{\mathrm{FD}} / \kappa_{A}=1.854$.

Neither of these results are good, but it is the kind of accuracy we should expect from a single minimal run. The power spectrum estimation in fact does better than the $g_{k}$ estimations in this case, but as we said, the procedure depends more heavily on manual control, which is usually not a good idea.

Now let us turn to case $\mathrm{C}$, whose conditions are completely identical to case B except changing the initial random number generator seed to have a different sequence. The goal is to find out how minimal runs differ from case to case. As it turns out, their difference can be quite large. Figure 13 shows the power spectrum of case $\mathrm{C}$, which does not seem very different from Figure 11. The $n \rightarrow 0$ limit gives a thermal conductivity value of $(1.7 \pm 0.4) \times 10^{7}$ at about $n=8$. However, the correlation function (Figure 14) behaves much better at small $k / L$ than case B. Applying the EF rule between $0.5 \leq k / L \leq 1.5$, we find that $L^{\prime} / L=0.778$, and the inferred thermal conductivity value is $\kappa_{C}^{\mathrm{EF}} / \kappa_{C}=0.972$. If we apply the FD rule, the FD in correlation function happens at $k_{\mathrm{dip}} / L=5.786$, and the FD estimation gives $\kappa_{A}^{\mathrm{FD}} / \kappa_{A}=1.123$. So in this case both results are better than the power spectrum estimation. This might be due to the fact the power spectrum in case $\mathrm{C}$ happens to fluctuate less at small frequency numbers (compare inset graphs of Figure 13 with Figure 11). 


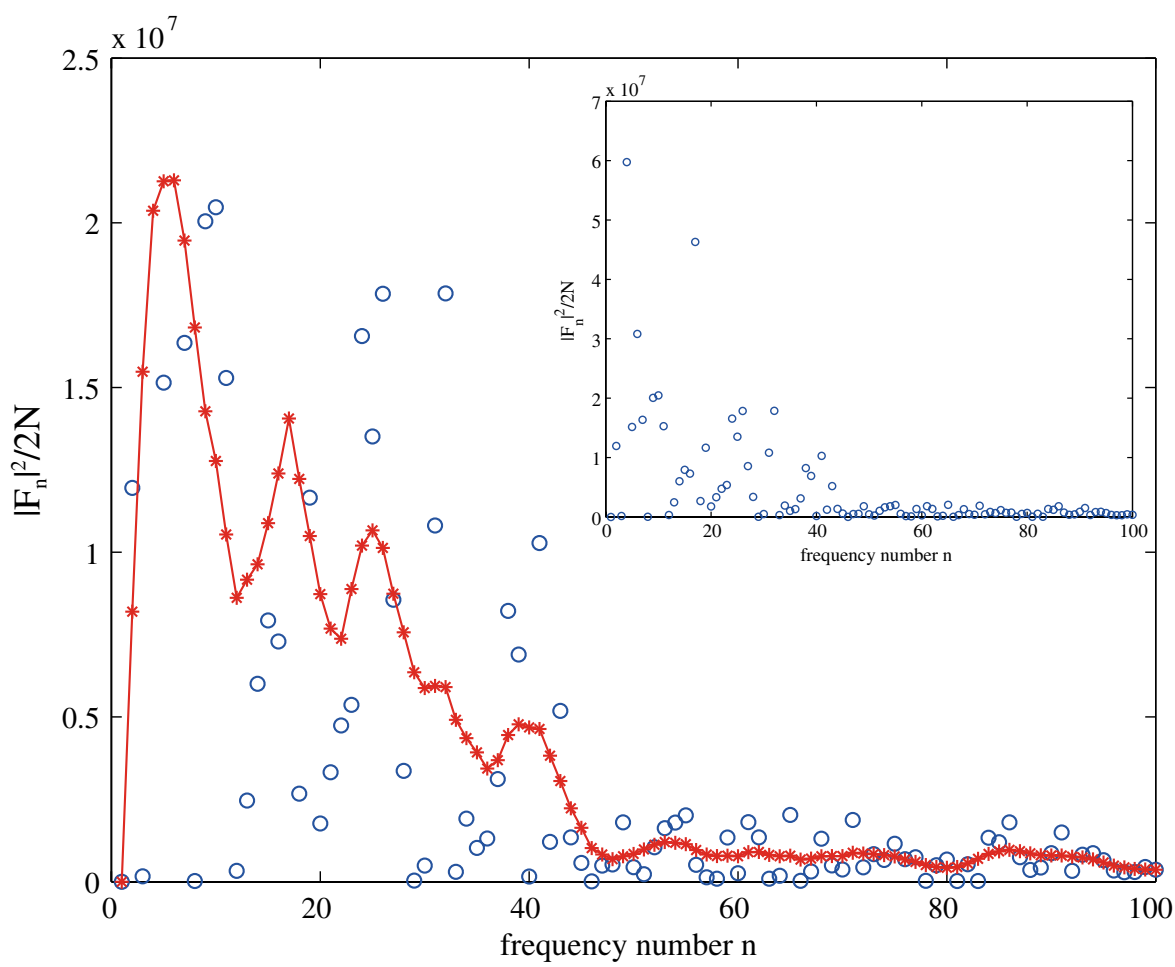

Figure 13. Power spectrum $\left|F_{n}\right|^{2} / 2 N$ versus frequency number $n$ in case C. The empty circles are the raw data, while the dots (with solid line to guide the eye) are the smoothed results after passing through a zero-phase filter of nearby 5 points. The inset graph shows the raw data on a larger scale.

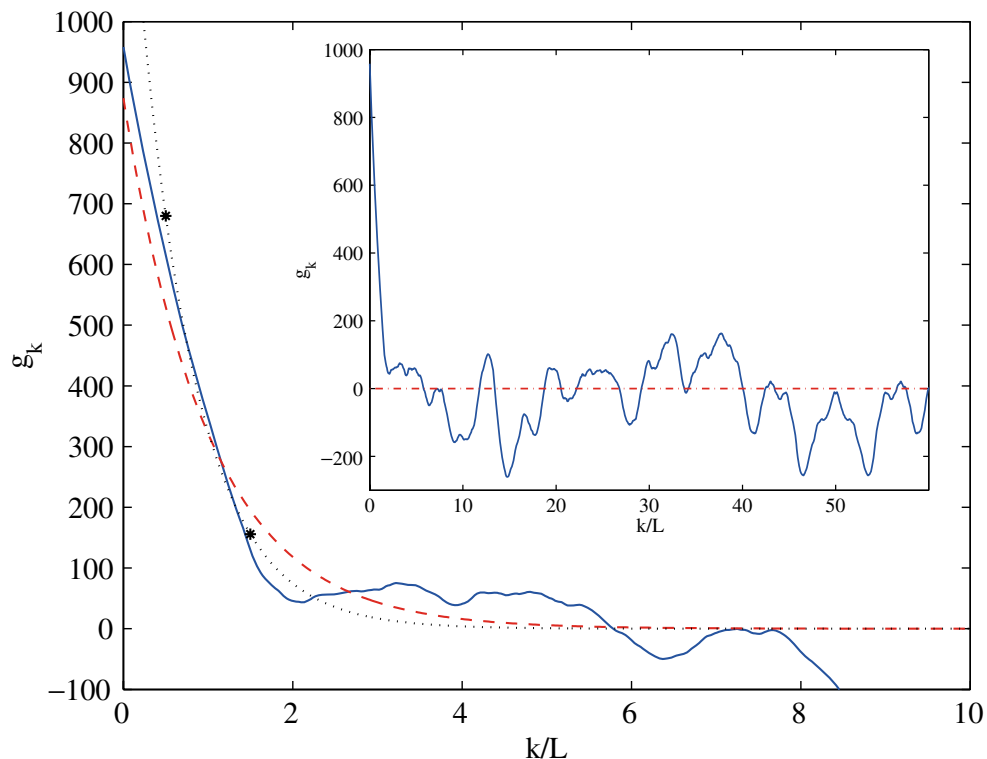

Figure 14. Correlation function $g_{k}$ versus $k / L$ in case C. The solid line is the numerical result; the dashed line is the theoretical prediction of Eq. (34). The dotted line is the EF fit to numerical result in the region $0.5 \leq k / L \leq 1.5$, indicated by the two dots. The inset graph shows the numerical result on a larger scale. 
Now we have a glimpse of the subtleties involved in calculating the thermal conductivity from minimal runs, which currently (circa 1998) are often the only things one can afford to do for realistic crystalline solids $(N / K \sim 30$ or $N / L \sim 100)$. On one hand, one expects the error to be large, say $50 \%$, and often biased to the positive side. On the other hand, some minimal runs may happen to hit right on target. In general, the difference from run to run is large. The difficulty is that unlike the present solvable model where one knows the exact answer, one cannot tell for sure which error is smaller. It is true that certain correlation curves 'look' better than others, such as Figure 14 than Figure 12, so one may assign larger weights to these results.

In the case of $\beta$-SiC, by making the assumption that the correlation function follows an exponential decay form, or that it never turns negative, we devise the EF and the FD termination rules. As we said in Section 4, those curves which give similar EF and FD values are often found to be closer to the correct (well-averaged) result. This is also found to be the case for our simple solvable model (compare case B and C); and it may also be helpful for other vibrational systems.

Notice that the EF results in case A, B and C are always better than the FD results, which we think is because the true correlation function is exponentially decaying. In general, we believe that the EF rule is more reliable for the data from a single minimal run, while the FD rule is better for well-converged or well-averaged data.

Should people do minimal-run simulations? Sometimes they are forced to: a minimal run for $\beta$-SiC at $T=500 \mathrm{~K}$ is 216 particles running 8 milllion steps under the Tersoff potential, which takes 50 processor hours on the high-end computer of SGI Origin 2000. The memory requirement to analyze the data afterwards is also demanding. But even if one can do very long simulations, one should investigate these issues before doing the runs. As we saw, case A has a much better convergence than case $\mathrm{B}$ and $\mathrm{C}$, but it takes (relatively) 10 times longer to run. We think that if one does 10 minimal runs $\left(N=2^{21} / 10\right)$ and average over the correlation functions, one will get comparable accuracy as case A. In reality one may benefit more from the latter route because one has taken a better ensemble average, by starting from different initial conditions (see the discussion in Section 1).

\section{Acknowledgements}

I thank Sidney Yip for support, discussions and encouragements during the research and writing of this manuscript. The development of the molecular dynamics code using the Tersoff potential was supported in part by AFOSR Grant No. F49620-96-1-0447. Acknowledgment is also made to the donors of The Petroleum Research Fund, administered by the ACS, for early support, and to Honda Motor Company for the donation of the SGI Origin 2000 computer, on which parts of the calculation were carried out.

\footnotetext{
Notes

${ }^{1} \mathrm{~g}(0)$ can be thought of as a static thermodynamic susceptibility which is usually not as sensitively dependent on the material state.
} 
2 There is some numerical difference between $K$ in Eq. (22) and $L$ in Eq. (28): $K$ signifies the maximum correlation length, above which correlation can be neglected, while $L$ is the characteristic period by which correlation decays by $e^{-1}$. If we consider $e^{-3} \approx 0.05$ to be 'negligibly small', then $K \approx 3 L$. For instance, in the $T=1500 \mathrm{~K} \beta$-SiC example in Section 4, the fitted EF decay time is $5.95 \mathrm{ps}(L)$, while we consider correlation to be significant up to $20 \mathrm{ps}(K)$ by looking at Figure 4 . So there should be $1 \ll n \ll N / 4 K \approx N / 12 L$.

\section{References}

1. Li, J., Porter, L. and Yip, S., J. Nucl. Mater. 255 (1998) 139.

2. Kubo, R., Rep. Prog. Phys. 29 (1968) 255.

3. Ladd, A.J.C., Moran, B. and Hoover, W.G., Phys. Rev. B 34 (1986) 5058.

4. Lee, Y.H., Biswas, R., Soukoulis, C.M., Wang, C.Z., Chan, C.T. and Ho, K.M., Phys. Rev. B 43 (1991) 6573.

5. Kitagawa, H., Shibutani, Y. and Ogata, S., Modell. Simul. Mater. Sci. Eng. 3 (1995) 521.

6. Kaburaki, H., Li, J. and Yip, S., Mater. Res. Soc. Symp. Proc. 538 (1999) 503.

7. Tersoff, J., Phys. Rev. Lett. 64 (1990) 1757.

8. Tersoff, J., Phys. Rev. B 49 (1994) 16349.

9. Press, W.H., Teukolsky, S.A., Vetterling, W.T. and Flannery, B.P., Numerical Recipes in C - the Art of Scientific Computing, Cambridge University Press, Cambridge, 1992. 